\title{
Spatial Resolution of Pre-reconstruction Raw Images and their Nano-CT Slices
}

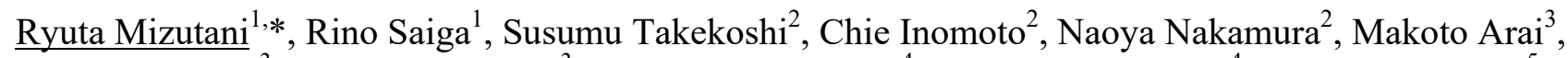
Kenichi Oshima ${ }^{3}$, Masanari Itokawa ${ }^{3}$, Vincent De Andrade ${ }^{4}$, Francesco De Carlo ${ }^{4}$, Akihisa Takeuchi ${ }^{5}$, Kentaro Uesugi ${ }^{5}$, Yasuko Terada ${ }^{5}$ and Yoshio Suzuki ${ }^{6}$

1. Department of Applied Biochemistry, Tokai University, Hiratsuka, Kanagawa 259-1292, Japan.

2. Tokai University School of Medicine, Isehara, Kanagawa 259-1193, Japan.

3. Tokyo Metropolitan Institute of Medical Science, Setagaya, Tokyo 156-8506, Japan.

4. Advanced Photon Source, Argonne National Laboratory, Lemont, IL 60439, USA.

5. Japan Synchrotron Radiation Research Institute (JASRI/SPring-8), Sayo, Hyogo 679-5198, Japan.

${ }^{6}$. Graduate School of Frontier Sciences, University of Tokyo, Kashiwa, Chiba 277-8561, Japan.

* Corresponding author, mizutanilaboratory@gmail.com

Image resolvability is the primary concern in microscopy. In micro/nano-tomography, a series of raw images are taken while rotating the sample. The resultant raw images are subjected to the tomographic reconstruction to visualize sample cross sections. Therefore, the resolution of the tomographic slice depends on the raw images. However, the resolutions of the pre-reconstruction raw images and their relationship with micro/nano-tomographic slices have not been delineated. Here, we report an analysis of the resolution of pre-reconstruction raw images of a human neuron and its reconstructed slices.

Post-mortem human brain tissues were collected with informed consent from the legal next of kin using protocols approved by ethical committees of the related organizations. Neurons in the brain tissue were labelled by Golgi impregnation [1]. The obtained tissue was embedded in epoxy resin. X-ray nanotomography was performed at the BL37XU beamline of SPring-8 (proposal nos. 2013A1384 and 2017A1143). A Fresnel zone plate with an outermost zone width of $50 \mathrm{~nm}$ was used as an x-ray objective lens, and an x-ray guide tube as a beam condenser [2]. The optics fluctuation was estimated to be less than $10 \mathrm{~nm}$. Absorption contrast images produced by $8-\mathrm{keV}$ x-rays were recorded using a CMOS-based imaging detector (AA60P and ORCA-Flash4.0, Hamamatsu Photonics). The effective pixel size was $23.0 \mathrm{~nm}$. A Siemens star chart with a minimum pitch of $100 \mathrm{~nm}$ was clearly resolved with these x-ray optics [3]. The tissue sample was mounted on a slide-guide rotation stage specially built for nanotomography (SPU-1A, Kohzu Precision). A total of 900 images were acquired with a rotation step of $0.20^{\circ}$ and exposure time of $800 \mathrm{~ms}$ per image.

Figure 1a shows a raw image of a human pyramidal neuron. The cell body apex indicated with a box was placed at the sample rotation axis so as to keep its position constant during the dataset acquisition. This box area was subjected to resolution estimation with the RecView program [4] available from https://mizutanilab.github.io/. Figure $1 \mathrm{~b}$ shows the resolution plot of Mizutani et al [3]. Point spread function (PSF) of region of interest (ROI) can be extracted using this plot independently of structures in the ROI [5]. The full width at half maximum of the PSF was estimated from the left end slope. Linear regression was performed by using data between $0.2 \times 10^{-5} \mathrm{~nm}^{-2}$ and $2.0 \times 10^{-5} \mathrm{~nm}^{-2}$. The obtained frame-by-frame resolutions (Figure 1c) showed a few jumps in the first half of the raw image dataset, suggesting that sample drift occurred during the image acquisition. The resolutions of the latter half showed a plateau indicating that sample deformation and x-ray irradiation reached some sort of equilibrium. The resolution fluctuations are also ascribable to other factors, such as optics instability or nanometer glitches of the rotation stage. Tomographic slices were reconstructed from this dataset. The 
resolutions of the slices were estimated and plotted against the position along the sample rotation axis (Figure 1d). The resolutions of the slices were mostly better than those of the raw images. This can be explained from the Hegerl-Hoppe theorem [6]. The resolution plot against the rotation axis showed no apparent trend, indicating that the resolution has no dependence on the position along the rotation axis.

This study revealed that the resolutions of raw images may fluctuate at the nanometer scale during data collection. The raw images and tomographic slices had slightly lower resolutions than that of the starchart image $(\leq 100 \mathrm{~nm})$. This suggests that rotation-stage precision and sample deformation, as well as the effect of the dose fraction theorem, are the resolution-determining factors of nanotomography.

[1] R Mizutani et al, Cerebral Cortex 20 (2010), 1739

[2] Y Suzuki et al, J Phys Conf Ser 463 (2013), 012028

[3] R Mizutani et al, J Microsc 261 (2016), 57

[4] R Mizutani et al, Nucl Instrum Meth A 621 (2010), 615

[5] R Saiga et al, Micron 105 (2018), 64

[6] R Hegerl and W Hoppe, Z Naturforsch 31a (1976), 1717

[7] This study was supported by grants from the JSPS (25282250 and 25610126).

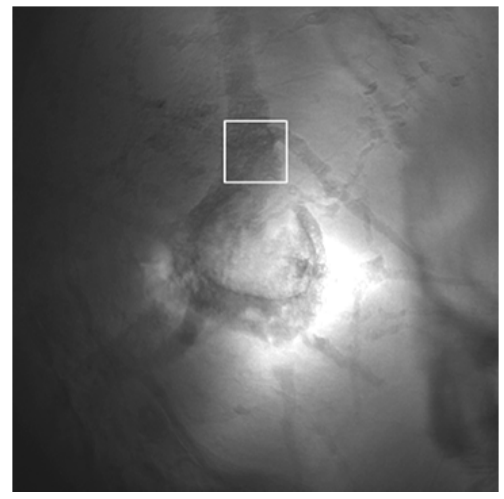

(a)

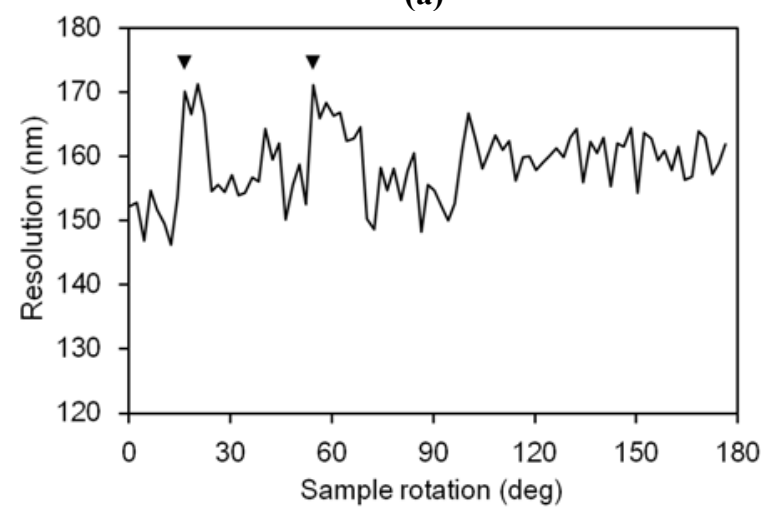

(c)

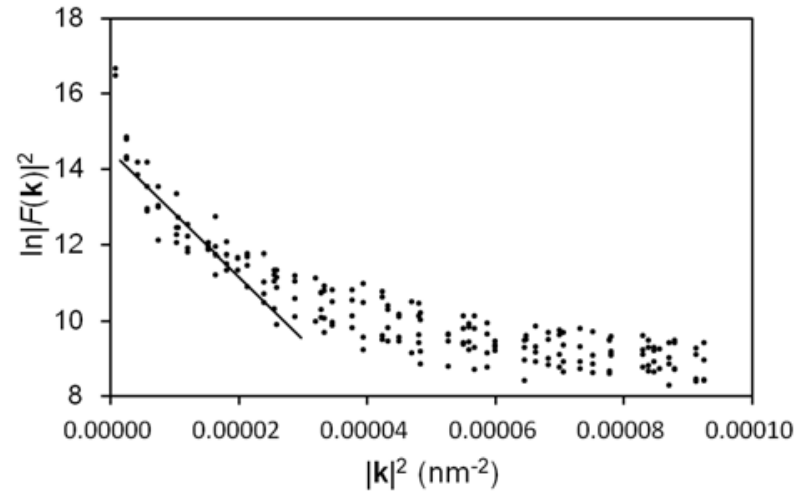

(b)

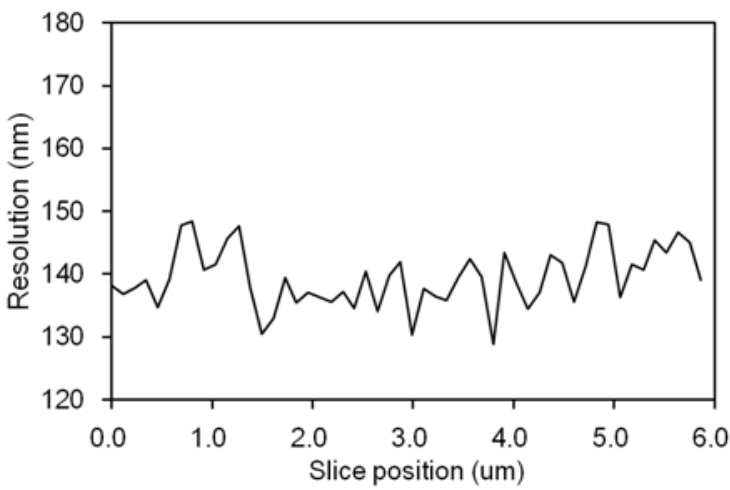

(d)

Figure. 1. (a) Raw image of a human pyramidal neuron. A $256 \times 256$ pixel $\left(5.9 \times 5.9 \mu \mathrm{m}^{2}\right)$ area of the cell body apex indicated with a white box was used for the resolution estimation. (b) Logarithmic plot of the boxed area. The line is a Gaussian approximation of the point spread function. (c) Resolutions of raw images estimated every 2 degrees of sample rotation. Triangles indicate possible sample drifts. (d) Resolutions of tomographic slices estimated every 5 slices. A $200 \times 200$ pixel region of the cross section of the cell body apex was used for the estimation. 